\title{
Comment on the brain areas whose blood supply is provided by the recurrent artery of Heubner
}

\author{
Ioannis Mavridis $\cdot$ Sophia Anagnostopoulou
}

Received: 5 June 2009 / Accepted: 3 August 2009 / Published online: 14 August 2009

(C) Springer-Verlag 2009

\section{Dear Editor,}

We have read with considerable interest the article by İzci et al. (published online on 4th February 2009) on the sulcal and gyral anatomy of the orbitofrontal cortex in relation to the recurrent artery of Heubner. They successfully reached the aim of their study to investigate the sulcal and the gyral anatomy of the orbitofrontal cortex with its arterial supply, providing a methodological approach to the numerous existing anatomic variations. We were also glad to read that the knowledge of the regional gyral and sulcal patterns in association with the arterial supply is crucial for a safe and efficient surgical procedure [2], because, in our opinion, this knowledge is often underestimated.

We would like to comment on the brain areas whose blood supply is provided by the recurrent artery of Heubner. İzci et al. reported that this artery supplies blood to important areas, such as the medial portion of orbitofrontal area, head of the caudate nucleus, anterior portion of putamen, external segment of globus pallidus, and anterior limb of the internal capsule [2]. We would just like to add another very important brain area where this important artery also supplies blood and which is the nucleus accumbens $[1,3]$. This nucleus is a major component of the ventral striatum and specifically the area of continuity between the head of the caudate nucleus and the putamen [5].

The recurrent artery of Heubner is the main, if not the only, artery supplying blood to this nucleus, which plays a crucial role in human behaviour and is also nowadays a target for deep brain stimulation in some carefully selected

I. Mavridis $(\bowtie) \cdot S$. Anagnostopoulou

Department of Anatomy, Medical School,

University of Athens, Mikras Asias 75,

Goudi, 11527 Athens, Greece

e-mail: pap-van@otenet.gr patients with refractory neuropsychiatric disorders. We have anatomically studied the branches of the A1 and A2 segments of the anterior cerebral artery and we have observed that its branch distributed at the nucleus accumbens' area is the recurrent artery of Heubner [Mavridis and Anagnostopoulou (2009) Unusual branches of the anterior cerebral artery found during an anatomical study of the blood supply of the human nucleus accumbens. Neurosurgical view. Submitted for publication]. An expected observation if we consider the other areas mentioned by İzci et al. to be supplied by this artery in combination with the location of the nucleus accumbens.

Finally, we agree with İzci et al. that the recurrent artery of Heubner is closely related to the gyrus rectus and that it demands special attention during anterior circulation aneurysm surgeries [2]. The neurosurgeon's ability to recognise and identify the anatomical variations of the recurrent artery of Heubner will reduce the occurrence of iatrogenic complications when performing surgery in and around the anterior half of the circle of Willis [4].

\section{References}

1. Feekes JA, Cassell MD (2006) The vascular supply of the functional compartments of the human striatum. Brain 129:21892201

2. İzci Y, Seçkin H, Medow J, Turnquist C, Başkaya MK (2009) Sulcal and gyral anatomy of the orbitofrontal cortex in relation to the recurrent artery of Heubner: an anatomical study. Surg Radiol Anat. doi:10.1007/s00276-009-0465-3

3. Kumral E, Evyapan D, Balkir K (1999) Acute caudate vascular lesions. Stroke 30:100-108

4. Loukas M, Louis RG, Childs RS (2006) Anatomical examination of the recurrent artery of Heubner. Clin Anat 19:25-31

5. Nolte J, Angevine JB Jr (2007) The human brain in photographs and diagrams, 3rd edn. Mosby (Elsevier), Philadelphia 